\title{
Volume of Distribution Steady State Observed by Fraction of Dose Absorbed
}

National Cancer Institute

\section{Source}

National Cancer Institute. Volume of Distribution Steady State Observed by Fraction of

Dose Absorbed. NCI Thesaurus. Code C156570.

The volume of distribution at steady state based on the observed CLST for a substance administered by extravascular dosing, divided by the fraction of dose absorbed. 